\title{
Avaliação de satisfação de usuários
}

\author{
Us er satisfaction assessment
}

Monique Esperidião 1

Leny Alves Bomfim Trad 1

1 Instituto de Saúde Coletiva da Un iversidade Federal da Bahia. Rua Basílio da Gama s/n, Campus Un iversitário, Canela, 40110-040, Sa lvador BA.

Abstract This study is a literatu re review with qualitative syntesis of the proeminent studies that analyses methodological issues of the user satisfaction with the health services. It was analyzed 54 articles present mostly in the databases Medline and Web of Science from 1970 to 2005. It is discussed the methodological approaches, techniques and instruments and the operacionalization of the concept satisfaction. Also, it is discussed different kinds of biases and ways of controlling them. It is observed that a large range of approaches can be identified, and there's little consensus among researchers. The most common approach is of quantitative nature, as questionnaires and scales. The qualitative approaches are valorized as the ones that can deal with the subjective nature of satisfaction. It is highly criticized the lack of standardization for the instruments, witch compromises the results. It is reflected the pertinence of the development of a proper approach to user satisfaction assessment in Brazil. Key words Patient satisfaction, Health service, Methods, Instruments
Resumo Es te estudo é uma revisão de literatura com sinte se qualitativa dos estudos voltados pa ra a análise metodológica das avaliações de satisfação de usuários com serviços de saúde. Foram analisados 54 artigos publicados principalmente em revistas indexadas pelo Medline e Web of Science no período de 1970 a 2005. São discutidos os métodos de abord a gem das pesquisas, as técn icas e instrumentos e a opera cionalização do conceito de satisfação. São apresentados, ainda, os diferen tes tipos de vieses das pesquisas e alternativas para sua superação. Ob serva-seque os métodos e técnicas variam em um largo escopo, havendo pouco consenso entre os pesquisadores. O método mais utilizado é de natureza quantitativa, com predominio de questionários e escalas. Os métodos qualitativos são valorizados como aqueles capazes de trabalhar os dados subjetivos da satisfação. Critica-sebastante a falta de padronização dos instrumentos, o que compromete a comparação dos achados. Reflete-se sob re a pertinência do desenvolvimen to de um referen cial próprio de pesquisa de satisfação para o Brasil.

Palavras-chave Satisfação de usuários, Serviços de saúde, Métodos, Instrumentos 


\section{Introdução}

A prá tica de avaliação dos serviços por meio da pergunta aos usuários difundiu-se a partir dos anos 60 na Eu ropa e EUA, inicialmente interes$s$ ada nos estu dos de adesão ao tratamento (Ware \& Snyder, 1975) e nas décadas de 1970 e 1980, inserida no movim en to da qualid ade dos serviços de saúde (Linder- Pelz, 1982; Turris, 2005). NoBrasil, os estu dos de satisfação ganham desta que a partir da segunda metade da década de 1990, com a promoção da accountability e o fortalecimen to da participação da comunidade nos processos de planejamento e avaliação. A literatura, então, tornou-se bastante ampla, compl exa e difusa (Aharony \& Strasser, 1993; Sitzia \& Wood, 1997).

Com efeito, a incorporação do usuário na avaliação tem sido valorizada não apenas por constituir-se um indicador sen sível da qualidade do serviço prestado, mas por estar potencialmente relacionada à maior adequação no uso do serviço (Trad et al., 2001). Aspectos ligados a mudanças de comport a m en to, incluindo adesão à orientação fornecida, podem ser iden tificados como resultantes do en ga ja men to do usuário como responsável pela avaliação (Ware et al., 1983; Weiss, 1988, Za s towny et al., 1989; Ah a rony \& Strasser, 1993; Bern hart et al., 1999; Ba ron-Epel et al., 2001; Trad et al., 2001; Joh a n s s on etal., 2002), o que articula, além do s aspectos econômicos de eficiência e eficácia dos serviços, a aceitabilidade social dos mesmos (Williams, 1994; Trad et al., 2001).

$\mathrm{Na}$ literatu ra sobre satisfação de usuários, é bastante peculiar observar que, a despeito de variabilidade de formas de se conceber e de medir a satisfação de usuários, a maioria dos estudos, en tre abordagens quantitativas e qualitativas, traz como resultado altas taxas de satisfação (Carr-Hill, 1992; Owens \& Bach el or, 1996; Avis et al., 1997; Souza, 1997). Es te fenômeno é conhecido na literatura como efei to de "el evação" das taxas de satisfação e é report ado mesmo quando as expectativas sobre os serviços são nega tivas. Tal fato tem levado os pesquis adores a questionar a validade de tais estudos, uma vez que, é pouco prová vel que os profissionais de saúde realizem sem pre um "cuidado perfeito" (Staniszerska \& Ahmed, 1999). E ra de se esperar que em países em desenvo lvimento, como o Brasil, por exemplo, on de o acesso a serviços de saúde de boa qualidade ainda é privilégio de poucos, os usuários manifestassem mais insatisfação com os serviços que lhes são oferecidos.
A falta de atenção a aspectos metodológicos das pesquisas de satisfação pode ser en tendida pelo fato de a satisfação de usuários representar um objeto de interesse apenas para geren tes e administradores preocupados em melhorar a qualidade dos serviços de saúde (Aha rony \& Strasser, 1993), sendo, de modo geral, desprezada pela comunidade "médica" e pela comunidade "científica" (Hudack \& Wright, 2000). Destaca-se ainda na litera tu ra um largo con s enso em considerar que a satisfação do pacien te é um con cei to po u co teorizado (Tu rris, 2005).

Parti n dodo baixo con s enso en tre pesquisadores sobre as diversas estrat égias metodológicas para a avaliação da satisfação de usuários e da possibilidade de vieses metodológi cos influenciarem o fenômeno da alta de satisfação, consideramos importante uma análise reflexiva da produção metodológica atualmente existen te. Nessa pers pectiva, o obj etivo de s te trabalho é apre sentar as con siderações metodológicas formuladas na literatura sobre a satisfação de usuários, evidenciando as principais abordagens disponíveis, discutindo seus limites e possibilidades.

Pa ratanto, foi conduzida uma revisão de literatura, com síntese qualitativa dos estudos voltados para a análise metodológica das avaliações de satisfação de usuários com serviços de saúde. Foram analisados 54 arti gos publicados em revistas index adas pelo Medline e Web of Science no período de 1970 a 2005. De modo complementar, visando capturar arti gos brasileiros, acessou-se as bases de dados LILACS. Pesquisas manuais também foram conduzidas a partir de listas de referências de outras revisões, buscando acessar artigos considerados importantes. Utilizaram-se como descri tores as palavras-chave: patient satisfaction, evaluation, health services, user satisfaction, methodology e revi ew combin ados de diferen tes form as.

\section{Métodos para a avaliação da satisfação de usuários}

Inicialmente vale considerar que, em bora seja referida a falta de consenso, a forma mais comum de con ceber o con cei to de satisfação é em termos de expectativas e da percepção que os u suá rios têm dos serviços recebi do s. De s temodo, o que se mede, muitas vezes, não é a satisfação propriamen te dita, mas a percepção dos serviços e a expectativa prévia dos usuários (Parasuraman, 1988; Vaitsman, 2003). 
Não raro, encon tramos os deb a tes acerca da questão metodológica debruçados sobre a natu reza dos métodos; qualitativos ou quantitativos. Esta preocupação também se reflete no campo da avaliação de satisfação de usuários. Os métodos quantitativos são os mais utilizados, especialmente os su rveys ou inquéri tos populacionais. In clu em a administração de questioná rios com questões fech adas e associam as questões a uma escala de valores, para men surar as respostas e qu a n tificar a satisfação (As p inal et al., 2003).

Os métodos quantitativos são valorizados ao permitirem alcançar uma amostra de usuários mais representativa da população, dificilmente obtida por outras metodologias. São t é cnicas consideradas de fácil manejo, pois, além de serem mais baratas, rápidas, podem ser administradas anonimamente, dispensando a presença de um pesqui s ador ou en trevi st ador trei$n$ ado quando o questionário é auto-aplicável. Em geral, utilizam-se escalas e questionários compostos de subitens que tratam de uma gama de questões incluin do estrutura, funcionamen to e avaliação da qualidade do serviço de saúde pelo usuário (Leichner \& Perrealut, 1990). Os quesitos dos questioná rios refletem o tipo de serviço em estu do e sua direção a um grupo específicode usuários.

Em con trapartida, os métodos quantitativos têm sido considerados reducionistas e sofrido críticas por não trabalharem os dados subjetivos do fenômeno da satisfação (Ah a rony \& Strasser, 1993; Avis et al, 1997; Owens \& Bachelor, 1996; Bosi \& Affonso, 1998; Coyle \& Williams, 2000; Dougall et al. , 2000; Trad et al., 2002). Em alternativa a esta abordagem, pesquisas qualitativas, incluindo as abordagens "compreensivas" ou "dialéticas" (Deslandes, 1997), perm i tem que os usuários falem sobre o serviço de saúde a partir de um leque mais amplo de posições. Dessa forma, fica mais explícito como um serviço se apresenta, ao mesmo tempo em que são focalizadas interações dos atores e contempladas considerações sobre c renças, modos de vida e con cepções do processo saúde-doença ( Trad et al., 2001). Owens \& Bachelor (1996) recomendam o uso de métodos qualitativos para pesquisa de satisfação quando se almeja alcançar grupos de difícil acesso, como grupos étnicos minoritários e pacientes idosos.

Aspinal et al. (2003) pontuam alguns limites nos métodos qualitativos. Para estes autores, entrevistas face a face costumam inibir a honestidade de pacientes e familiares por medo de sof rer represálias, além de con sumir bastante tem po para que se estabeleça um rapport entre pesquisador e usuários, sendo cansativo para pacien tes debilitados. O utro aspecto a ser considerado rem ete à questão da alterid ade na pesquisa qualitativa. De fato, de acordo com Min ayo \& Sanches (1993), uma vez que a pesquisa qualitativa promove uma aproximação entre sujeito e objeto, de natureza comum, é preciso diferenciar a compreensão que se tem do outro e da realidade, da compreensão intro s pectiva de si mesmo.

O utro questionamento comumen te fei to a pesquisas qualitativas é sobre a representatividade da fala indivi dual em relação ao coletivo. A esse respeito, gostaríamos de com entar que, ao con trá rio do que se possa imaginar, embora as técnicas qualitativas se valham de uma a m o stra reduzida de sujeitos, elas não produzem, necessariamente, m edidas exclu s iva m en te individuais. Teóri cos sociais têm mostrado que falas individuais são tecidas de material ideológico; são tramas de todas as relações sociais e acompanham as mudanças sociais de um grupo. Sendo assim, em apenas uma fala está con tida toda a representação de grupos determinados em condições históricas, socioeconômicas e culturais específicas (Mi n ayo \& Sanches, 1993).

Uma com binação de métodos também tem sido sugerida para o estudo da satisfação de usuários, supera n do os limites associados a cada um deles em particular (Aspinal et al., 2003). Assim, se permite que sejam privilegiados espaços para análises quantitativas, buscando captar a amplitude do fenômeno; e qualitativas, que permitam aprofundar sua complexidade. É referi do que as duas abord a gens de pesquisa não se en con tram em situação de oposição, e sim de continuidade e complementaridade (Min ayo \& Sanches, 1993; Serpa, 2002).

Outro deb a te travado na literatura discute os modos de en dereçamento das questões rel ativas à satisfação, entre a abordagem direta ou indireta. Embora Hall \& Dornan (1988) apontem que ambos os métodos são confiáveis, a lgumas con siderações têm sido levantadas para c ada ti po. As questões diretas perguntam explicitamen te sobre níveis de satisfação com ex periências dos serviços como em: você ficou satisfeito com os serviços recebidos? Na maioria das vezes, as perguntas diretas são criticadas por produzirem níveis de satisfação geral, ao lidarem com apenas uma dimensão da satisfação (La Monica et al., 1986; Ca rr-Hill, 1992). 
$\mathrm{Na}$ abordagem indireta, os pesquisadores ou avaliadores inferem os níveis de satisfação a partir de questões dirigidas ao serviço provido. São mais relacionadas a estudos que consideram aspectos multicausais da satisfação (La Monica et al., 1986; Carr-Hill, 1992; Fakhoury et al., 1996; Coyle \& Williams, 2000). In dependen temente do modo em que são en dereçadas as questões, Williams (1994) sinaliza que as dicotômicas limitam as possibilidades de respostas dos usuários, forçando a avaliação dos serviços em termos de satisfação/insatisfação, impedindo a ex pressão de sentim en tos ambíguos e/ou não dicotômicos.

Pesquisadores avaliaram a variação da satisfação no tem po e no local on de é medida, no serviço de saúde ou no domicílio. As pesquisas realizadas na unidade de saúde, após a consulta, buscam resgatar a experiência concreta do usuário no serviço, evitando efeitos de perda de memória. Kloetzel et al. (1998) mediram a satisfação logo após a consulta e depois de 10 dias da con sulta (em casa e no serviço), en contrando para ambos os casos os mesmos resultados. É rel a tadona literatura o efei to de "satisfação tardia", que se relaciona mais com os resultados do atendimen to e refere-se aos casos cujo desfecho clínico é positivo. A satisfação medida logo após a con sulta costuma estar associada a aspectos da relação médico-paciente, em especial a informação dada a respeito da doença e seu tratamen to (Jack son et al., 2001). Alguns pesquisadores também comentam que o indivíduo ten de a ser mais críti co com o passar do tem po. À medida que se tem a ex pecta tiva superada, novas expectativas são formadas, e de modo mais exigente. Ainda como possívd explicação, conforme Jack s on et al. (2001), com o passar do tem po é mais prová vel que o usuário recorde-se de momentos desagradáveis, pois são even tos que costumam ser mais marcantes do pon to de vista cognitivo.

\section{Instrumen tos e técnicas para a avaliação da satisfação}

Estudos de revisão mais antigos já questionavam a validade e a confiabilidade dos instrumen tos (Lebow, 1974; Rubim, 1989). Na revisão metodológica de Rubim (1989), nenhum in s trumen to en con trado na literatu ra apresent ava os critérios de validade e de confiabilidade adotados por este autor. Pou cos estu dos compararam os escores da satisfação en con trados entre os usuários com outras medidas, como a percepção de profissionais ou familiares $(\mathrm{Ru}-$ bim, 1989).

Há po u co con senso sobre a escolha dos instrumen tos de medida da satisfação. Numerosos instrumen tos podem ser identificados na literatura e a maioria dos trabalhos utiliza mais de um instrum en to (Aspinal et al., 2003). As técnicas e instrumen tos de pesquisa variam em um largo escopo: entre caixas de sugestões, linhas telefônicas, sistemas de vídeo, inquéritos transversais, entrevistas, observações, grupos focais e estudos qualita tivos de abord a gem antropológica (Ka toka et al., 1997; Souza \& Pereira, 1999). As três primeiras abord agens apresentam muitas limitações, consti tu in do-se apenas em um primei ro en foque, pouco sofisticado, para a aproximação do tema a fim de ori entar trabalhos futuros. O sistema de vídeo, embora produzindo medidas de fidedignidade, não são considerados adequ ado s, pois, além de invadirem a privacidade do médico e do paciente, induzem o "efei to de Hawth orne" - em que médicos e pacien tes modificam seus comportamentos por estarem sendo avaliados (Souza \& Perei ra, 1999). Ademais, es te método permite apenas con h ecer a relação médico - p aci ente no setting da con sulta.

Segundo Souza (1997), a maioria dos instrumentos não foi testada em outros sistemas de saúde, de modo que muitos dos itens de investigação não são transferíveis ou adaptáveis às condições de outros serviços ou de outros sistemas de saúde. Os países que "importam" métodos de pesquisa podem com prom eter a valid adee confiabilidade de sua avaliação, caso não façam uma adequ ada ad aptação transcultural.

A falta de padronização entre os instrumen tos den tro de um mesmo país costuma ser referida por diversos autores no campo, dificultando a com $\mathrm{p}$ a ração dos re sult ados (LinderPelz, 1982; Hall \& Dornan, 1988; Ross et al., 1993; Lewis, 1994). O estudo de meta-análise de Hall \& Dornan (1988), con du zi do com uma amostra de 221 estudos, mostrou que $97 \%$ dos instrum en tos utilizados eram elaborados pelo próprio pesquisador. Apenas 3\% já eram conhecidos e haviam sido utilizados em outras pesquisas. Os instrumentos são assim elaborados numa "produção casei ra", os cha mados homemade questionnarie (Lewis, 1994).

A elaboração das escalas para medição do grau de satisfação é um fator importante a se considerar quando da elaboração de um instrumen to. As escalas servem para mensurar a 
resposta obtida, a tribuin do pesos e valores para cada item. A maioria das escalas é baseada no modelo de Likert, a bord a n do a satisfação de modo indireto pela con cordância ou discordância com as afirmativas sobre o serviço ou s obre o profissional, a partir de categorias que variam entre: "concordo plenamen te" e "discordo plenamen te". De acordo com a revisão de Aspinal et al. (2003), as escalas usadas não são escalas de satisfação propriamente ditas, mas instrumentos psicométricos ou escalas de sintomas ou de qualidadedevida, em bora os resultados sejam descritos em termos de satisfação.

Em relação às técnicas qualitativas, os grupos focais têm sido con si derados essenciais para a obtenção de dados qualitativos necessários pa ra a con tex tualização cultu ral do nível de satisfação do usu á rio com o serviço avali ado (Edwards \& Staniszewska, 2000; Carr-Hill, 1992; Trad et al. , 2001; 2002; Schi der \& Palman, 2002). Nesta técnica, um gru po rela tivamen te homogên eo é convidado a discutir um dado tópico da avaliação. O gru po é guiado por um facilitador e um moderador, a discussão é gravada em fita cassete/vi deo teipe. A técnica de gru pos focais tem sido valorizada por constituir-se uma estratégia de coleta de informações de caráter qualitativo em profundidade, em curto espaço de tempo e a baixo custo (Stewart \& Shamdasani, 1990; Green baum, 1998; Gomes \& Ba rbosa, 1999). São especialmente interessantes em situações nas quais se deseja ampliar a compreensão de um determinado proj eto, programa ou serviço, tal qual as pesquisas de avaliação de satisfação.

Uma varian te deste modelo é o processo de gru po nominal (Carr-Hill, 1992). Nesta técnica, os participantes são questionados pelos entrevistadores e suas respostas são escritas em um pedaço de papel, independen temen te do gru po. Depois, as respostas são lidas e discutidas em uma nova rodada de questões e respostas. Esta técnica é bastante usada quando o repertório de re spostas é mui to diver gen te sobre um dado aspecto do serviço avaliado en tre um mesmo grupo de usuários (Carr-Hill, 1992). Vale destacar que, para o obj etivo da avaliação da satisfação de usuário, a técnica não está direcionada para a busca de um con s enso en tre o gru po, uma vez que a pluralidade de respostas espelha a diversidade de experiências relativas a um serviço. Mas ajuda o pesquisador a confirmar uma determinada informação que consi dere importante e deve ser destac ada en tre as falas dos usuários.

\section{Principais vieses e altern a tivas para investigar as razões da alta satisfação en contradas nas investigações}

Alguns pesquisadores têm buscado com preender o fenômeno da alta satisfação. Enquan to uns apontam inconsistências teóricas no conceito de satisfação (Williams, 1994; Sitzia \& Wood, 1997; Williams et al., 1998), o utros o relacionam a aspectos metodológicos (Wa re et al., 1983; Leichner \& Perrealut, 1990). No caso dos serviços públicos, argumenta-se ainda que a alta satisfação pode expressar o receio dos usuários em perder o direito ao serviço, mesmo sendo este de baixa qualidade, fa zendo do usuário do sistema público uma espécie de usuário cativo (Sobrinho, 1996; Gomes et al., 1999).

Em relação aos aspectos metodológicos, questiona-se a sensibilidade dos métodos em discriminar pacientes satis fei tos e insatisfeitos e quan to à apropriação das dimensões selecionadas para o estudo da satisfação (Carr-Hill, 1992). A alta satisfação pode repres entar a expressão das técnicas em pregadas na obtenção da informação como o tipo de entrevistador e o modo pelo qual as perguntas são formuladas e en dereçadas.

Os estudos diferem na escolha do instrumen to, na valoração dos ach ados, na definição do mom en to de abordar o usuário, na iden tificação e no con trole dos principais vieses. Uma crítica recorren te está na ausência de padron ização dos instrumentos, reduzindo a possibilidade de comparação entre as pesquisas. A maioria dos pesquisadores do tema desenvolveu seus próprios instrumentos de medida, mas não descreve quais procedimentos adotaram (Leichner \& Perrealut, 1990), o que dificulta a avaliação sobre a eficácia dos mesmos.

Visando desenvo lver uma metodologia para a avaliação da qualidade dos serviços ambulatoriais, Kl oet zel et al. (1998) te st a ram quatro versões de um mesmo questionário para grupos distin tos de usuários de um mesmo serviço. Embora a sen sibilid ade dos métodos variasse, a comparação dos result ados mostrava altíssima con cordância en tre as respostas: todos demonstravam alta de satisfação. Do ponto de vista metodológico, alguns fatores têm sido apontados como responsáveis pelo fenômeno da alta satisfação, caso, por exemplo, da relutância em ex pressar opiniões nega tivas con hecido como o viés de gratidão - gratitude bias (Owens \& Bach el or, 1996; Avis et al., 1997; Bernhart et al., 1999) e o viés de aquiescência, re- 
lacion adb à enu m eração dos que si tos dos qu e stionários (Locker \& Dunt, 1978).

A aquiescência costuma revelar-se nos instrumentos que dispõem de escala. Trata-se da tendência do indivíduo em concordar com o prim ei ro item da escala indepen den tem en te do s eu con teúdo. Portanto, pode influenciar o nível de satisfação na ordem dos itens, ou seja, para mais, caso os itens estejam ordenados positivamente, ou para menos, na situação inversa (Ross et al. , 1993). Essa tendência é mais evidenciada entre os usuários mais idosos e nos indivíduos com menor renda (Ware, 1975; Fitzpatrick, 1991, Ross et al., 1993).

Outro tipo de viés identificado é o viés de gratidão (gratitude bias). Cos tuma ser en contrado em países em de senvo lvimen to. Trata-se da omissão de questionamen tos e críticas negativas dos usuários, e é verificado especialmen te na avaliação de serviços públi cos. Mas, o gratitude bias pode aparecer em situações em que o usuário tem grande afinidade com os profissionais provedores do cuidado, como em casos de pacientes internos. Pode também refletir uma postura de gratidão fren te ao pesquisador. Neste caso, a medida de satisfação aparece distorcida, pois é, por si só, uma fonte de satisfação para este (Bern ha rt et al., 1999).

Alguns autores apontam que a mídia, sob o a s pecto de form adora de opinião pública, pode ser identificada como uma fonte de viés. No Brasil, habitualmente, os diversos conjun tos de mídia propagam uma idéia de serviço público de sprovido de qualidade. Essa difusão muitas vezes é feita de forma explícita e agressiva (So uza, 1997). No entanto, este fenômeno deveria influ enciar os result ados dos estu dos de satisfação de modo a produzir insatisfação. A maioria das pe squisas aponta o con trário.

Uma prová vel explicação para esta questão reside na observação de Gilbert et al. (2000) que a mídia influ encia mais a opinião de não-usu ários do que a de usu á ri o s. Seguindo este rac i ocínio, a diferença de opinião en tre usu á rios e nãousuários pode re sultar de uma antiga e persistente impressão de que o serviço público não funciona, ao passo que tu do o que é privado é de qualidade. Há outras explicações possíveis, como a satisfação dos usu á rios pelo simples fato de ter a ga ran tia da prestação dos serviços. Outro el em en to que pode influ enciar as percepções dos serviços do setor públ i coé a confiança dos cidadãos no govern o, nos políticos e nos servidores públicos. A percepção quanto à hon esti$\mathrm{d}$ ade e à integridade do governo afeta a avalia- ção do públ i co em relação aos serviços que recebem dessas insti tuições (Santos, 1995).

Pa ra con tornar o fen ô m eno do "exagero" de satisfação, a utores vêm desenvo lven do estratégias de manejo metodológico. Katoka et al. (1997) dest acam a importância de se compararem as re spostas fechadas e abertas do questionário, pois é comum o usuário atribuir uma avaliação alta nas respostas fechadas e, nas a bertas, in cluir com entários, restrições e recomendações sobre o mesmo item. Contudo, os autores chamam a atenção para a necessidade de análise do conteúdo das observações feitas quando da comparação entre as respostas fech adas e abertas. A con tradição apontada pelo usuário pode descrever, na verd ade, uma contradição do serviço oferecido (Kotaka et al., 1997).

Silva et al. (1995) analisam que quando a tendência é registrar a satisfação (em seus aspectos positivos), os resultados desfavoráveis en contrados (insatisfação) devem ser registrados com atenção, pois se tornam indicadores relevan tes sobre o serviço em questão. Alguns estu dos passaram a destacar a importância de se debruçar sobre a avaliação da "insatisfação" (Wa re et al., 1983; Zastowny et al., 1989; Coyle, 1999), pois esta categoria permite revelar aspectos dos serviços que precisam ser melh orados e, assim, interessam aos administradores (Carr-Hill, 1992).

Williams (1994) considera que, antes mesmo de se avaliar a satisfação do usuário com o serviço, é preciso enten der quais os mecanismos que o mesmo utiliza para avaliar o serviço em questão, ou seja, entender o processo cognitivo do usuário envolvido na avaliação. Aponta também a necessidade de se buscar no ideário do usuário o significado do termo satisfação, além dos aspectos que levam à satisfação e insatisfação.

Os instrumen tos de satisfação geral, ou índ i ces de satisfação global, também são tidos como possíveis propiciadores do fenômeno da "alta de satisfação". Lei ch n er\& Perrealut (1990) a pontam que o uso do índice de satisfação geral, no qual somen te um ou pou cos itens mensu ram a satisfação, n em mesmo uma análise fatorial permite discriminar as distintas dimensões envolvidas. Com vistas a recuperar as lacunas deixadas pelas medidas gerais provenientes de tais instrumentos, alguns pesquisadores têm desenvo lvi do instrum en tos multidimensionais da satisfação (Leichner \& Perre alut, 1990). Estes permitem discriminar as distintas 
dimensões da satisfação, assegurando uma maior com preensão da apreciação dos serviços na ótica dos usuários a partir de cada uma das dim ensões da satisfação explorada, possibilitando a ação com maior precisão nos fatores que devem ser melhorados.

Vale lem brar que alguns autores com entam que uma gama de questões tem sido abordada nos questionários e escalas indistintamente, inclu in do a satisfação com o aten dimen to na recepção, a alimentação fornecida, limpeza do quarto, roupa de cama e aspectos interpessoais, acessibilidade, en tre outros. Is to pode refletir que a noção de satisfação é multidimensional e, portanto, sua mensuração deve ser também multifacetada. Mas pode indicar, ainda, que os pesquisadores não têm cla reza do que estão medindo.

\section{A operacionalização do con cei to de satisfação: aspectos dos serviços a serem avaliados}

A falta de uma definição con sis ten te da satisfação e de uma teoria que oriente a sua mensuração é refletida na diversidade de abordagens m etodológicas adotada pelos estudos. Há diferenças importantes na operacionalização do con cei to de satisfação, ou seja, na definição de seus com pon en tes ou dimensões e no modo de medi-la (Ware \& Snyder, 1975; Williams \& Calnan, 1991). As dimensões da satisfação são aspectos dos serviços tais como ace sso, qualidade, estrutura física e organizacional, aspectos da relação médico-paciente, entre outros. A escolha destas dimensões condiciona e determina o de senvo lvimento de parâmetros e critérios utilizados na seleção dos métodos. A avaliação da satisfação difere a depender do número e exatidão com que a performance de tais compon entes é explorada (Accurcio et al., 1991).

Diversas classificações das dimensões da satisfação têm sido pospostas, algumas apropriadas apenas para alguns tipos de serviços de saúde, outras buscandoum modelo mais a bra ngen te (Sitzia \& Wood, 1997). A taxonomia mais aceita en tre as pe squisas foi elaborada por Ware et al. (1983), distinguindo oito dimensões: 1) com portamento interpess oal (modo em que provedores intera gem com os pacientes: respeito, preocupação, amizade, cortesia);2) qualidade técnica do cuidado (competência e aderência a altos critérios de diagnósti co e tratamento; 3 ) acessibilidade/conveniência (tempo de espera, facilidade de acesso); 4) aspectos financeiros; 5) eficácia/resultados do cuidado (melhoria e manutenção da saúde); 6) continuidade do cuidado; 7) ambien te físico (ambi en tes bem sinalizado s, equipamentos, a tmosfera agradável); 8) disponibilidade (presença de recursos médicos: quantidade suficiente de provedores e insumos).

Prévost et al. (1998) desenvolveram uma classificação mais ampla, privil egi an doasatisfação nas seguintes dimen sões: rel acional (respeito, consideração, acolhida, gentileza, etc.); organizacional (or ganização dos serviços); profissional (tempo dispen s ado na consulta, in formações suficientes e claras, etc.); e socioeconômicos e culturais (aspectos relacionados às condições de vida das pessoas). Trad et al. (2002), avaliandoa satisfação de usuá rios com o Programa de Saúde da Família, adicionou ao modelo de Prévost et at. (1998) a dimensão cognitiva, destacando a con cepção dos usuários (en ten dim en tosobre a lógica de funcionamen to) do programa.

A comunicação entre os profissionais de saúde e os usuários (cla reza das informações, explicação sobre o diagnósti co e tratamen to, esclarecimento de dúvidas e disponibilidade para contato em situações emergenciais) tem ganhado relativa autonomia entre as dimensões da satisfação, sendo privilegiada na mensuração da satisfação (Rubim, 1989; Sitzia \& Wood, 1997; Williams et al., 1998).

Por fim, é fundamental destacar que o contex tobrasileiro indica outros fatores rel evantes, como a estrutura e organização do sistema de serviços de saúde e modelos assistenciais vi gentes. Sem dúvida, o acesso é um atributo dos serviços bastante valori zado pelos usuários. Geralmen te o usuário de serviços públi cos faz parte de populações que vivem em condições soc i oeconômicas precárias e que buscam os serviços de forma assistencial (para o atendimen to médico e busca de medicam en tos e vacinas). Estes usuários interessam-se mais por um padrão mínimo de sobrevivência do que pela própria qualidade dos serviços (Santos, 1995).

\section{Considerações finais}

Diantedesta revisão, observa-se que os diferentes métodos, técnicas e instrumentos apresentam vantagens e desvantagens, não haven do ainda para a avaliação da satisfação estratégias metodológicas válidas e confiáveis que sejam de con s enso dos pesquisadores. 
Ne s tes termos, pensamos que uma combinação de técnicas é uma estratégia bastante oportuna para dar conta da complexidade da satisfação e evitar reducionismos presen tes em cada método. Vale ainda destacar a importância da triangulação de dados, investindo na contraposição da avaliação realizada pelos usuários com a pers pectiva dos profissionais e/ou ges tores dos serviços, no sen tido de buscar uma maior contextualização dos dados obtidos.

Embora exista um conjunto de métodos e técnicas que possuem um grau de universalidade, há que se considerar as diferentes peculiaridades de cada país. Autores naci onais vêm refletindo que as pesquisas sobre satisfação do usuário no Brasil não devem representar apenas um acréscimo "copiado" de metodologia importada dos países cen trais (Oliveira, 1991; Lemme et al., 1991; Serapione, 1999). Dada a diferença entre o padrão socioeconômico e cultural da população que utiliza os serviços, exis teanecessidade de confecção de um instrum ental adequ ado a nossa realidade. As pesquisas no Brasil devem refletir, especialmente, $\mathrm{di}$ ficuldades nas condições de acesso aos serviços de saúde e devem estar adequadas ao perfil de baixa escolaridade e consciência cívica de seu público(Vaitsman et al., 2003).

Remarca-se ainda a distinção entre os serviços de saúde de natu reza pública e privada, e as características particulares de suas dientelas. Nos serviços públicos, o poder de pressão dos usuários é baixo, pois não há necessidade de captação de clien tela, como no setor privado. As avaliações de satisfação de usuário de serviços públ i cos podem repre sentar um importante meio para con tornar esta questão, atuando como instrumentos de dar voz aos usuários (voice giving). De s temodo, perm i tem aos usuários oportunidades de ex pressão nas quais podem monitorar e controlar as atividades dos serviços públicos de saúde, fortalecendo sua participação nos processos de planejamento e exercen do con trole social.

Por fim, a de spei to da necessidade de aperfeiçoamento de instrumentos e métodos de pesquisa no âmbi to da satisfação de usuários, vale lembrar que esse movimen to deve estar orient ado para uma avaliação con seqüente, cujo result ado tra ga aperfeiç oa men to para o cotidiano dos serviços de saúde, propician doavanços significa tivos no âmbito da gestão dos serviços de saúde.

\section{Colaboradores}


Referências bibliográficas

Accurcio FA, Cherchilia ML \& Santos MA 1991. Avaliação de qualidade de serviços de saúde. Saúde em Deba te 33:50-53.

Aharony L \& Strasser S 1993. Patient satisfaction: what we know about and what we still need to explore. Medical Care Revi ew 50(1):49-79.

Aspinal F, Addington-Hall, Hughes R \& Higginson IJ 2003. Using satisfaction to measure the quality of palliative care: a review of the literature. Journal of Advanced Nu rsing42(4):324-339.

Avis M, Bond M \& Arthur A 1997. Questioning patient satisfadion: an em p i rical inve s tiga tion in two outpati ent dinics. Jou rnal of Advanced Nu rsisng 44(1):8592.

Baron-Epel O, Dushenat M \& Friedman N 2001. Evaluati on of the consumer model: relashionship bet ween patient's expectations, perception and satisfaction with care. International Jou mal for Quality in Health Care 13(4):317-323.

Bernhart MH, Wiad nyana IGP, Wihardjo H \& Pohan I 1999. Pati ent satisfaction in devel oping countries. Social Scien ceand Medicine 48:989-996.

Bosi ML \& Af fonso KC 1998. Participação popular e saúde: com a palavra, os usu á rios da rede pública de serviços. Caderno de Saúde Pública 14(2):355-365.

Calnan M 1988. Towards a con ceptual fra mework of lay evalua tion of health care. Social Science and Medicine 27(9):927-933.

Ca rr-Hill RA 1992. The measu rement of patient satisfaction. Jou rnal of Pu blic Health Medicine 14(3):236-249.

Coyle J 1999. Exploring the meaning of "dissatisfaction" with health care: the import a n ce of "pers onal iden tity thre a t". Sociology of Health \& Illness 21(1):95-123.

Coyle J \& Wi lliams B 2000. An exploration of the epistemological intricacies of using qualitative data to devel op a qu a n titative measu re of user views of health care. Journal of Advanced Nu rsing31:1235-1243.

Dougall A, Russell A, Ru bim G \& Ling J 2000. Rethinking patient satisfaction: pati ent experiences of an open access flexible sigmoidoscopy service. Social Science and Medicine 50:53-62.

Deslandes SF 1997. Concepções em pesquisa social: articulações com o campo da avaliação em serviços de saúde. Caderno de Saúde Pública 13(1):103-107.

Edwards C \& Staniszewska S 2000. Accessing the user's perspective. Health \& Social Care in the Community 8(6):417-424.

Fakhoury et al. 1996. Determinants of in formal caregivers' sati sfacti on with servi ces for dying cancer patients. Social Scien ceand Medicine 41:721-731.

Fitzpatri ck R 1991. Surveys of patient satis faction: I - Important gen eral considerations. Brith. Medical Journal 302:1129-1132.

Gilbert RG, Nicholls JAF \& RoslowS 2000. A men suração da satisfação dos cl i en tes do setor públi co Revista do Serviço Público51(3):26-40.

Gomes R, da Si lva CMF, Deslandes SF \& Souza ER 1999. Avaliação da assistência ambulatorial a portadores de HIV/AIDS no Rio de Ja n ei ro, segun do a visão dos usuários. Cademo de Saúde Pública 15(4):789-797.

Gomes MES \& Barbosa EF 1999. A técnica de gru pos focais para obtenção de dados qualitativos. Revista Educa tiva, São Paulo.
Greenbaum TL 1998. The Handbook for Focus Group Research. S AGE Publications, Thousand Oaks.

Hall JA, Dornan MC 1988. Meta-analysis of satisfaction with medical care: description of research domain and analysis of overall satisfaction levels. Social Scien ce and Medicine 27(6):637-644.

Hudak PL \& Wright JG 2000. The characteristics of pati ent satisfacti on measures. Spine 25(24):3167-3177.

Jack s on JL, Cja mberlin J \& Kroenke K 2001. Predictor of patient satisfaction. Social Science and Medicine 52:609-620.

Joahansson P, Oléni M \& Fridlund B 2002. Patient satisfacti on with nu rsing care in the con text of the health care: a literatu re stu dy. No rdic Coll ege of Caring Sciences, Scand J. Caring Sci 16:337-344.

Kotaka F, Pach eco MLR \& Higaki 1997. Avaliação pelos usuários dos hospitais participantes do programa de qualidade hospitalar no Estado de São Paulo, Brasil. Revista de Saúde Pública 31(2):171-177.

Kloetzel K, Bertoni AM, Irazoqui MC, Campos VPG \& Sa n tos RN 1998. Con trole de qualidade em atenção primária à saúde. I - A satisfação do usuário. Caderno de Saúde Pública 14(3):623-628.

La Monica EL, Oberts MT, Madea AR \& Wolf RM 1986. Developm ent of a pati ent satisfadi on scale. Rese arch in Nursing and Health 9:43-50.

Lebow JL 1974. Consumer assessments of the quality of medical care. Medical Care 12(4):328-337.

Leichner P \& Perrealut M 1990. Developpmente et validation d'une echelle multi-dimensionelle de satisfaction de patients de services d'hospitalisation en psychiatrie Rapport Final. Société Canadienne d'Évaluation, Montréal.

Lemme AC, Noronha G \& Resen de JB 1991. A satisfação do usu á rio em hospital universitário. Revista de Saúde Pública 25(1):41-46.

Lewis JR 1994. Patient views on quality care in general practice: litera tu re revi ew. Social Science and Medicine 39(5):655-670.

Linder- Pelz S 1982. Tow a rd a theory of pati ent satisfaction. Social Scien ceand Medicine 16:577-582.

Locker D \& Dunt D 1978. Theoretical and methodologi$\mathrm{cal}$ issues in sociological studies of consumer satisfacti on with medical care. Social Science and Medicine 12(4):283-292.

Minayo MCS, Deslandes SF, Cruz Neto O \& Gomes R 2001. Pesquisa social: teoria, método e criatividade. Vozes, Petrópolis.

Min ayo MCS \& Sanches O 1993. Quantita tivo-qualitativo: Oposição ou complementaridade? Caderno de Saúde Pública 9(3):239-262.

Owens DJ \& Bach el or C 1996. Patient satisfacti on and the el derly. Social Science and Medicine 42(11):14831491.

Oliveira CG 1991. B reve análise da catego ria de sat isfação na obra de Donabedian. Dissertação de mestrado. In stituto de Medicina Social, Centro Biomédico, Un iversid adedo Estado do Rio de Jan eiro, Rio de Ja neiro.

Parasumaran A, Zeithaml V \& Berry L 1988. A multipleitem scale for measuring customer perceptions of servicequality. Journal of Retailing 12-40.

Pr évost A, FafardA \& Nadeau MA 1998. La mesure de la satisfaction des usagers dans le domaine de la santé 
et des services sociaux: l'experience de la Régie Régi onale Chadière-Appalaches. The Canadian Journal of Pro gram Evaluation 13(1):1-23.

Ross CK, Steward CA \& Sinacope JM 1993. The importance of pati ent preferen ces in the measu rement of health care satisfaction. Medical Care 31(12):11381149.

Rubim HR 1989. Pati ent evaluati ons of hospital care: a revi ew of li terature. Medical Care 28(9):53-59.

San tos MP 1995. Avaliação da qualidade dos serviços públicos de atenção à saúde da criança sob a ótica do usuário. Dissertação de mestrado. Instituto de Sa ú de Coletiva, Un iversidade Federal da Bahia, Sa lvador.

Schider H\& Palman N 2002. Get ting to the truth? Researching user views of primary health care. Health Policy and Planning 17(1):32-41.

Serapione M 1999. Avaliação da qualidade em saúde: a con tri buição da sociologia da saúde para a su peração da polarização en tre a visão dos usu á rios e a pers pectiva dos profissionais de saúde. Saúde em Debate 23(53):81-92.

Serpa LF 2002. Qualidade-quantidade na pesquisa educacional. Ágere - Revista de Educação e Cultura 6(6): 165-174.

Si lva LMV, Formigli VLA, Cerquei ra MP, Kruchevsky L, Teixei ra MMA, Ba rbosa ASM, Con ceição PSA, Khouri MA \& Nascimento CL 1995. O processo de distritalização e a utilização de serviços de saúde - avaliação do caso de Pau da Lima. Caderno de Saúde Pública 11(1):72-84.

Sitzia J \& Wood N 1997. Pati ent satisfaction: A review of issues and concepts. Social Science and Medicine 45(12):1829-1843.Sobrinho CLN 1996. Municipalização das ações de saúde: a experiência de SantaluzBahia - 1993/1995. Dissertação de mestrado. In stitur to de Sa ú de Col etiva, Universidade Federal daBahia, Sa lvador.

Souza EM 1997. A sati sfação dos idosos com os serviços de saúde: um estudo de prevalência e de fato res associados em Taguatinga, DF. Dissertação de mestrado. Faculdade de Ciências da Saúde, Universidade de Brasília. Brasília.

Souza EM \& Pereira M 1999. A satisfação do usuário na avaliação dos serviços de saúde. B rasília Médica 36 (1/2):33-36.

S tewart DW \& Shamdasani P 1990. Focus group research: exploration and discovery. SAGE Publications, Newburry Park.
Staniszenska S \& Ah m ed L 1999. The con cepts of ex pectation and satisfaction: do they capture the ways patients evalu a tetheir care? Jou rnal of Advanced Nu rsing 29(2):364-372.

Trad LA B, Ba stos ACS, Santana EM \& Nunes M 2001. A construção social da estratégia de saúde da família: condições, su jei tos e contexto s. PNEPG, CNPq.

Trad LA B, Ba stos ACS, Santana EM \& Nunes M 2002. O estudo etn ográfico da satisfação de usuário do Programa de Sa ú de da Família (PSF) na Bahia. Ciên ciae Saúde Col etiva 7(3):581-589.

Turris AS 2005. Un packing the con cept of patient satisfaction: a feminist analysis. Journal Advanced Nu rsing 50 (3): 293-298.

Vaitsman J, Andrade GRB, Farias LO, Médici ME \& Nascimento LR 2003. Projeto Gestão de Qualidade e Satisfação dos Usuários em Organizações Públicas de Saúde. Um estudo piloto no Instituto de Pesquisa Evandro Chagas - IPEC. Re sumo do relatório final apresentado à Fundação de Amparo à Pesquisa do Rio de Ja n ei ro-FAPERJ. ENSP/Fiocruz.

Ware JE \& Snyder MK 1975. Dimensions of patient attitu des rega rding doctors and medical care services. Medical Care 13:669.

Ware JE, Snyder MK, Wright WR \& Davies AR 1983. Defining and measuring patient satisfaction with medical care. Evaluation and Program Planning 6:247263.

Wartman AS, Morlock LL, Malitz FE \& Palm AE 1983. Patient understanding and satisfaction as predictors of compliance. Medical Care 21:386-391.

Weiss GL 1988. Pa ti ent satisfaction with primarymedical care: evaluati on of sociodemographic and pred is positional factor. Medical Care 1988; 26(4):383-392.

Williams B 1994. Pati ent satis faction: a valid con cept? Social Science and Med i cine38(4):509-551.

Williams B, Coyle J \& Healy D 1998. The meaning of patient satisfaction: An explanation of high reported l evels. Social Scien ce \& Med i cine47(9):1351-1359.

Williams SJ \& Calnan M 1991. Key determinants of consumer satisfaction with general practice. Journal of FamilyPractice 8:237-242.

Williams B, Weinman J \& Dale J 1998. Doctor-patient communication and patient satisfaction: a review. Family Practice 15(5):480-492.

Zastowny TR, Roghmann KJ \& Cafferata GL 1989. Patient satisfaction and the use of Health Services: Explora ti oninCausality. Medical Care27(7):705-723.

Artigo apresen t ado em 30/04/2004

Aprovado em 5/06/2005

Versão final apresen tada em 20/09/2005 